\title{
Upregulation of alveolar neutrophil enzymes and long pentraxin-3 in human chronic lung allograft dysfunction subtypes
}

\begin{abstract}
Tomohito Saito, MD, PhD, ${ }^{\mathrm{a}, \mathrm{b}}$ Mingyao Liu, MD, MSc, ${ }^{\mathrm{a}, \mathrm{c}}$ Matthew Binnie, MD, ${ }^{\mathrm{d}}$ Tereza Martinu, MD, ${ }^{\mathrm{d}}$ Masaaki Sato, MD, PhD, ${ }^{\mathrm{a}, \mathrm{e}}$ and Shaf Keshavjee, MD, MSc, ${ }^{\mathrm{a}, \mathrm{c}}$ Toronto, Ontario, Canada, and Osaka and Tokyo, Japan
\end{abstract}

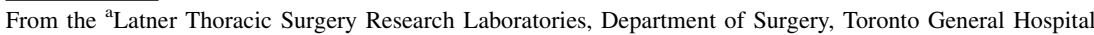
Research Institute, University Health Network, and ${ }^{\mathrm{c} I n s t i t u t e}$ of Medical Science, Faculty of Medicine, and ${ }^{\mathrm{d}}$ Division of Respirology, University of Toronto, Toronto, Ontario, Canada; the ${ }^{\mathrm{b}}$ Department of Thoracic Surgery, Kansai Medical University, Hirakata, Osaka, Japan; and ${ }^{\mathrm{e}}$ Department of Thoracic Surgery, University of Tokyo Graduate School of Medicine, Hongo, Tokyo, Japan.

Disclosures: Authors have nothing to disclose with regard to commercial support.

Supported by the Canadian Institutes of Health Research (MOP-190953), Canadian Cystic Fibrosis Foundation (grant 2387), and Ontario Ministry of Research and Innovation (grant GL2-01-019). T.S. was supported by Research Fellowships of the Japan Society for Promotion of Science for Young Scientists.

Received for publication Dec 26, 2017; accepted for publication Feb 17, 2018; available ahead of print March 21, 2018.

Address for reprints: Shaf Keshavjee, MD, MSc, Toronto General Hospital, 200 Elizabeth St, 9N946, Toronto, Ontario M5G 2C4, Canada (E-mail: shaf.keshavjee@uhn.ca).

J Thorac Cardiovasc Surg 2018;155:2774-6

$0022-5223 / \$ 36.00$

Copyright $(c) 2018$ by The American Association for Thoracic Surgery

https://doi.org/10.1016/j.jtcvs.2018.02.039
}

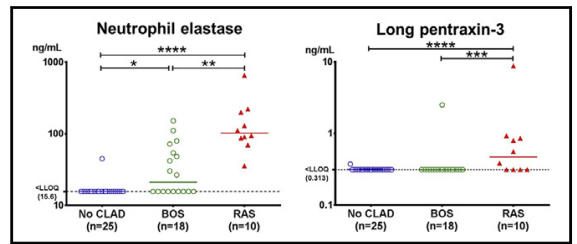

Upregulated neutrophil elastase and long pentraxin-3 in restrictive allograft syndrome.

\section{Central Message}

Activation of innate immunity may play a key role in the development of post-lung transplant restrictive allograft syndrome, an aggressive form of chronic lung allograft dysfunction.

See Editorial Commentary page 2777.
Supplemental material is available online.

Chronic lung allograft dysfunction (CLAD) has been a major challenge to long-term success after lung transplantation. To date, 2 distinct CLAD subtypes are noted: restrictive allograft syndrome (RAS) and bronchiolitis obliterans syndrome (BOS). RAS progresses rapidly, demonstrating pleuroparenchymal fibroelastosis, often admixed with diffuse alveolar damage and obliterative bronchiolitis, whereas BOS shows slower progression with obliterative bronchiolitis as major lesion. Our recent study demonstrated further upregulation of alveolar S100 family proteins and high-mobility group box 1 in RAS relative to $\mathrm{BOS},{ }^{1}$ indicating an important role of innate immunity in the development of RAS. To discriminate the biologic characteristics of RAS and BOS, we investigated human CLAD by profiling alveolar expressions of innate immune components, such as neutrophil elastase (NE), $\alpha$-defensins, matrix metalloproteinase (MMP)-8, and long pentraxin-3 (PTX3), in bronchoalveolar lavage (BAL) fluid.

\section{METHODS AND RESULTS}

This study was approved by the University Health Network Research Ethics Board (REB\#11-0506-T). Written consent for the use of BAL samples was obtained from each patient in adherence to the principles set forth in the Declaration of Helsinki. BAL samples from 28 patients with CLAD (10 patients with RAS and 18 with BOS) and 25 samples from subjects without CLAD (no CLAD group) were analyzed. One sample from the RAS group was unavailable for measurement of $\alpha$-defensins and MMP-8. These samples were used in our previous alveolar alarmin profiling study. ${ }^{1}$ Appendix E1 in the online for this report contains details on study population; BAL fluid collection; definitions of CLAD, RAS, and BOS; standard posttransplant care; measurement and evaluation of alveolar proteins; and statistical analysis. Clinical and demographic characteristics of the study population and concurrent pathologic and microbiologic findings in the BAL samples were described in our previous study. ${ }^{1}$ The lower limit of quantification (LLOQ) was defined as the lowest calibration standard of the assay kits.

Alveolar expressions of NE, $\alpha$-defensins, MMP-8, and PTX3 are summarized in Figure 1. The RAS group showed significantly upregulated NE, $\alpha$-defensins, MMP-8, and PTX3 relative to the no CLAD group (NE median, 

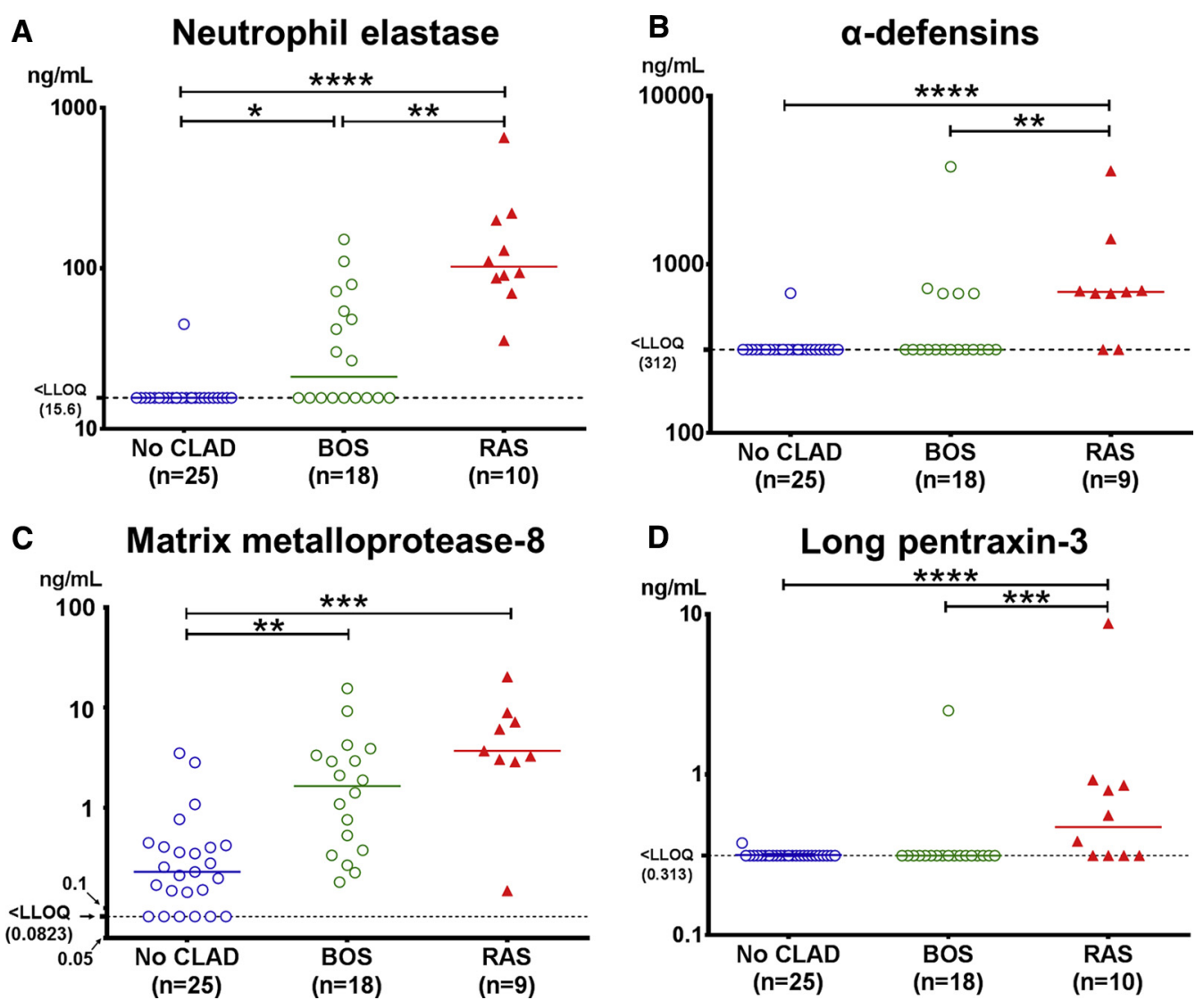

FIGURE 1. Protein expressions of alveolar neutrophil elastase (A), $\alpha$-defensins (B), matrix metalloproteinase- 8 (C), and long pentraxin-3 (D). The restrictive allograft syndrome $(R A S)$ group showed significantly increased alveolar expression of neutrophil elastase, $\alpha$-defensins, matrix metalloproteinase-8, and long pentraxin-3 relative to the no chronic lung allograft dysfunction (No CLAD) group. The bronchiolitis obliterans syndrome (BOS) group also showed upregulation of alveolar neutrophil elastase and matrix metalloproteinase-8 relative to the no chronic lung allograft dysfunction group (A and C). Moreover, the restrictive allograft syndrome group showed further upregulation of neutrophil elastase, $\alpha$-defensins and long pentraxin- 3 compared to bronchiolitis obliterans syndrome. The lower limit of quantification $(L L O Q)$ was defined as the lowest calibration standard of the assay kit (described in detail in Appendix E1). $P$ values are as follows: Asterisk indicates $P<.05$; double asterisk indicates $P<.01$; Triple asterisk indicates $P<.001$; and quadruple asterisk indicates $P<.0001$.

$102.5 \mathrm{ng} / \mathrm{mL}$ vs LLOQ; $P<.0001 ; \alpha$-defensins median, $696.8 \mathrm{pg} / \mathrm{mL}$ vs LLOQ; $P<.0001 ;$ MMP-8 median, $3.70 \mathrm{ng} / \mathrm{mL}$ vs $0.23 \mathrm{ng} / \mathrm{mL} ; P=.0002 ;$ PTX3 median, $0.471 \mathrm{ng} / \mathrm{mL}$ vs LLOQ; $P<.0001)$. The BOS group showed upregulated NE and MMP-8 relative to the no CLAD group (NE median, $21.1 \mathrm{ng} / \mathrm{mL}$ vs LLOQ; $P=.024$; MMP-8 median, $1.65 \mathrm{ng} / \mathrm{mL}$ vs $0.23 \mathrm{ng} / \mathrm{mL} ; P=.002$ ).

Moreover, the RAS group showed further upregulation of NE, $\alpha$-,defensins and PTX3 relative to the BOS group (NE median, $102.5 \mathrm{ng} / \mathrm{mL}$ vs $21.1 \mathrm{ng} / \mathrm{mL} ; P=.0027 ; \alpha$-defensins median, $696.8 \mathrm{pg} / \mathrm{mL}$ vs LLOQ; $P=.008$; PTX3 median, $0.471 \mathrm{ng} / \mathrm{mL}$ vs LLOQ; $P=.0004)$. In differentiating RAS from BOS, NE, $\alpha$-defensins, and PTX3 showed high to moderate accuracy (NE area under the curve, 0.894; 95\% confidence interval, $0.777-1.012 ; P=.0007 ; \alpha$-defensins area under the curve, $0.784 ; 95 \%$ confidence interval, $0.583-0.985 ; P=.018$; PTX3 area under the curve, 0.761 ; $95 \%$ confidence interval, $0.555-0.967 ; P=.024)$.

\section{DISCUSSION}

We demonstrated that alveolar expressions, as measured by enzyme-linked immunosorbent assay, of NE, $\alpha$-defensins, and PTX3 were almost undetectable in BAL fluid in the no CLAD group but significantly upregulated in the RAS group, and even significantly higher than in the BOS group. The differentiation of RAS from BOS has been challenged by the lack of specific biomarkers. These 3 molecules, especially PTX3, which was detected almost exclusively in RAS group samples, could be candidates for further studies. On the other hand, MMP-8 was upregulated in both BOS and RAS forms of CLAD.

$\mathrm{NE}$ and $\alpha$-defensins, both released from primary granules of neutrophils, may contribute to alveolar damage in RAS through the degradation of extracellular matrix. ${ }^{2}$ MMPs derive primarily from tertiary granules of neutrophils, and MMP-8, also known as neutrophil collagenase, may contribute to extracellular matrix remodeling in both RAS 
and BOS. ${ }^{2}$ PTX3, a soluble pattern recognition receptor that is rapidly produced by phagocytes and resident cells at the site of inflammation, recognizes pathogens and eliminates them through complement activation and opsonization. ${ }^{3}$ In addition, PTX3 is stored in secondary granules of neutrophils and is a component of neutrophil extracellular traps (NETs) ${ }^{4}$ which entrap and kill pathogens. BAL samples from RAS patients showed upregulation of other NET components, NE and high-mobility group box 1 (a suggested inducer of NETs). ${ }^{4,5}$ Massive formation of NETs and increased neutrophil degranulation can be detrimental to the host, and the overactivation of these innate immune responses may be involved in the pathogenesis of RAS.

We recognize important limitations of this study. These include small sample size, unavailability of standardized quantitative data regarding the volume of recovered BAL samples, and lack of matching of immunosuppressive agents between the no CLAD group and both CLAD groups. ${ }^{1}$

\section{CONCLUSIONS}

Our results identified upregulation of alveolar NE, $\alpha$-defensins, and PTX3 in RAS relative to BOS, further supporting the contention that activation of innate immunity may play an important role in the development of the more aggressive form of CLAD, RAS. Further refinements in the biologic profiling may contribute to the better understanding of the specific pathologic mechanisms underlying the CLAD subtypes and the development of precisely targeted therapy for each.

We thank Jerome Valero for his assistance in editing the manuscript, David Hwang for his help in verification of pathologic diagnosis, Sassan Azad and Paul Chartrand for their contribution toward facilitating this study, and Sarah E. Gilpin, Thomas K. Waddell, and Marcelo Cypel for their support for this study. We also thank Naoki Minato and Yukihito Saito for their mentorship of T.S.

\section{References}

1. Saito T, Liu M, Binnie M, Sato M, Hwang D, Azad S, et al. Distinct expression patterns of alveolar "alarmins" in subtypes of chronic lung allograft dysfunction. Am J Transplant. 2014; 14:1425-32.

2. Kennedy VE, Todd JL, Palmer SM. Bronchoalveolar lavage as a tool to predict, diagnose and understand bronchiolitis obliterans syndrome. Am J Transplant. 2013;13:552-61.

3. Daigo K, Inforzato A, Barajon I, Garlanda C, Bottazzi B, Meri S, et al. Pentraxins in the activation and regulation of innate immunity. Immunol Rev. 2016;274: 202-17.

4. Delgado-Rizo V, Martínez-Guzmán MA, Iñiguez-Gutierrez L, GarcíaOrozco A, Alvarado-Navarro A, Fafutis-Morris M. Neutrophil extracellular traps and its implications in inflammation: an overview. Front Immunol. 2016;8:81

5. Tadie JM, Bae HB, Jiang S, Park DW, Bell CP, Yang H, et al. HMGB1 promotes extracellular trap formation through interactions with Toll-like receptor 4. Am J Physiol Lung Cell Mol Physiol. 2013;304:L342-9. 


\section{APPENDIX E1. SUPPORTING INFORMATION: SUPPLEMENTAL MATERIAL TO METHODS SECTION \\ Study Population}

Bronchoalveolar lavage (BAL) samples banked in the Toronto Lung Transplant Program Biobank were selected if the post-bilateral lung or post-heart-lung transplant survivor had chronic lung allograft dysfunction (CLAD) develop before undergoing BAL. ${ }^{1}$ Specimens were excluded if the patient had overlapping proven or probable pulmonary infection at the time of BAL, in compliance with the International Society for Heart and Lung Transplantation consensus statement. ${ }^{\mathrm{E} 1}$ In total, $28 \mathrm{BAL}$ samples from 28 patients with CLAD (10 with restrictive allograft syndrome [RAS] and 18 with bronchiolitis obliterans syndrome [BOS]) were included. In addition, we included 25 BAL samples from 25 post-bilateral lung or post-heartlung transplant recipients with stable lung function as a "no CLAD" control group, matching the interval from lung transplantation to BAL collection with those of the RAS and BOS groups.

\section{Posttransplant Follow-up, Immunosuppression, and Treatment for CLAD}

BAL fluid and transbronchial biopsy collection were planned at 2 and 6 weeks after lung transplant; at 3, 6, 9, 12,18 , and 24 months after lung transplant; and when clinically indicated. Our protocol for initial standard immunosuppression regimen included cyclosporine (INN ciclosporin), azathioprine, and prednisone. ${ }^{\mathrm{E} 2}$ In brief, cyclosporine was converted to tacrolimus in patients who had cyclosporine toxicity or development of recurrent acute rejection with adequate cyclosporine levels. Mycophenolic acid or mycophenolate mofetil was used in patients with anti-HLA antibodies, patients who did not tolerate azathioprine, and patients who had recurrent rejection while receiving azathioprine. When CLAD developed, patients were routinely switched from cyclosporine to tacrolimus, and thrice weekly azithromycin was implemented. A replacement of azathioprine with mycophenolic acid was also considered.

\section{Collection of BAL Fluid}

BAL fluid was collected after 2 repeat lavages of $50 \mathrm{~mL}$ of normal saline solution in the right middle lobe or the lingula in lung transplant recipients. ${ }^{\mathrm{E} 3}$ Bronchoscopies with BAL fluid collections were routinely performed at 2 and 6 posttransplant weeks and then every 3 months for the first year, every 6 months for the second year, and as clinically indicated. The quantity of recovered BAL fluid was greater than $30 \mathrm{~mL}$ of $100 \mathrm{~mL}$-instillation in all 53 subjects, except for 1 patient with RAS. Aliquots of the BAL fluid were collected and immediately snap frozen at $-80^{\circ} \mathrm{C}$. After thawing, protease inhibitors (Complete Mini Tabs; Boehringer-Mannheim, Mannheim, Germany) were added to the samples, which were then clarified by centrifugation at $5000 \mathrm{~g}$ for 10 minutes. The resulting supernatant was assayed for protein measurement.

Definitions of CLAD, RAS, and BOS. The baseline forced expiratory volume in 1 second $\left(\mathrm{FEV}_{1}\right)$ was defined according to criteria of the International Society for Heart and Lung Transplantation, ${ }^{\mathrm{E} 4}$ and the baseline total lung capacity (TLC) value was calculated as the average of the parameters measured at the time of the best $\mathrm{FEV}_{1}$ measurements. CLAD was defined as an irreversible decline in $\mathrm{FEV}_{1}$ below $80 \%$ of baseline ${ }^{\mathrm{E} 5}$ BOS was strictly defined as CLAD without restrictive changes of RAS. RAS was defined as CLAD with an irreversible decline in TLC below $90 \%$ of baseline. To exclude RAS, the diagnosis of BOS was thus not made until a valid TLC measurement had been collected. The diagnosis of RAS was not made until the $\mathrm{FEV}_{1}$ dropped to meet the criteria for CLAD, even if TLC had already declined to meet the threshold. The diagnosis of CLAD was made only if functional decline persisted after appropriate treatment for infection, acute rejection, or both. Declines in $\mathrm{FEV}_{1}$ and TLC were considered reproducible only when 2 separate measurements at least 3 weeks apart met the thresholds for CLAD and RAS, respectively. The first date of decline in $\mathrm{FEV}_{1}$ and TLC that met the criterion for each condition was recorded as the onset date.

\section{Measurement and Evaluation of Protein Expressions}

We measured protein expressions of neutrophil elastase (NE), $\alpha$-defensins, matrix metalloproteinase (MMP) 8 , and long pentraxin 3 (PTX3) in BAL supernatant by means of enzyme-linked immunosorbent assay kits (NE and MMP8; Abcam, Cambridge, UK; $\alpha$-defensins/human neutrophil peptide 1-3; Hycult Biotech, Uden, Netherlands; PTX3, Quantikine; R\&D System, Minneapolis, Minn) according to manufacturer instructions. BAL supernatants were diluted 100 -fold for NE, 2000-fold for $\alpha$-defensin, and 10-fold for MMP-8 quantification. No dilution was applied for PTX3. Lower limit of quantification (LLOQ) was defined as the lowest calibration standard of the assay kits ${ }^{\mathrm{E} 6, \mathrm{E} 7}$ : $0.156 \mathrm{ng} / \mathrm{mL}, 156 \mathrm{pg} / \mathrm{mL}, 8.23 \mathrm{pg} / \mathrm{mL}$, and $0.313 \mathrm{ng} / \mathrm{mL}$ for NE, $\alpha$-defensins, MMP-8, and PTX3, respectively.

\section{Statistical Analysis}

The Kruskal-Wallis test was used to determine differences among patients with RAS, BOS, and no CLAD. Values below LLOQ were substituted with the value of the LLOQ described in the Methods section. To report diagnostic accuracy in differentiating CLAD from No CLAD, or RAS from BOS, receiver-operating characteristic curves were constructed and the area under the curve was calculated. GraphPad Prism version 6.02 (GraphPad Software, San Diego, Calif) for Windows (Microsoft, Redmond, Wash) was used for additional analyses. 


\section{E-References}

E1. Husain S, Mooney ML, Danziger-Isakov L, Mattner F, Singh N, Avery R, et al. A 2010 working formulation for the standardization of definitions of infections in cardiothoracic transplant recipients. J Heart Lung Transplant. 2011;30:361-74.

E2. de Perrot M, Chaparro C, McRae K, Waddell TK, Hadjiliadis D, Singer LG, et al. Twenty-year experience of lung transplantation at a single center: Influence of recipient diagnosis on long-term survival. J Thorac Cardiovasc Surg. 2004; 127:1493-501.

E3. D’Ovidio F, Mura M, Ridsdale R, Takahashi H, Waddell TK, Hutcheon M, et al. The effect of reflux and bile acid aspiration on the lung allograft and its surfactant and innate immunity molecules SP-A and SP-D. Am J Transplant. 2006;6:1930-8.

E4. Estenne M, Maurer JR, Boehler A, Egan JJ, Frost A, Hertz M, et al. Bronchiolitis obliterans syndrome 2001: an update of the diagnostic criteria. J Heart Lung Transplant. 2002;21:297-310
E5. Sato M, Waddell TK, Wagnetz U, Roberts HC, Hwang DM, Haroon A, et al. Restrictive allograft syndrome (RAS): a novel form of chronic lung allograft dysfunction. J Heart Lung Transplant. 2011;30:735-42

E6. U.S. Department of Health and Human Services, Food and Drug Administration, Center for Drug Evaluation and Research, Center for Veterinary Medicine. Guidance for industry: bioanalytical method validation; 2001. Available at: https:// www.fda.gov/downloads/Drugs/GuidanceCompilanceRegulatoryInformation/ Guidances/ucm070107.pdf.

E7. European Medicines Agency, Committee for Medicinal Products for Human Use, Efficacy Working Party. Guideline on bioanalytical method validation; 2011. Available at: http://www.ema.europa.eu/docs/en_GB/ document_library/Scientific_guideline/2011/08/WC500109686.pdf. 